\title{
Toward a geometric analogue of Dirichlet's unit theorem
}

\author{
Atsushi Moriwaki
}

\begin{abstract}
In this article, we propose a geometric analogue of Dirichlet's unit theorem on arithmetic varieties; that is, if $X$ is a normal projective variety over a finite field and $D$ is a pseudo-effective $\mathbb{Q}$-Cartier divisor on $X$, does it follow that $D$ is $\mathbb{Q}$-effective? We also give affirmative answers on an abelian variety and a projective bundle over a curve.
\end{abstract}

\section{Introduction}

Let $K$ be a number field, and let $O_{K}$ be the ring of integers in $K$. Let $K(\mathbb{C})$ be the set of all embeddings $K \hookrightarrow \mathbb{C}$. For $\sigma \in K(\mathbb{C})$, the complex conjugation of $\sigma$ is denoted by $\bar{\sigma}$; that is, $\bar{\sigma}(x)=\overline{\sigma(x)}(x \in K)$. Here we define $\Xi_{K}$ and $\Xi_{K}^{0}$ to be

$$
\left\{\begin{array}{l}
\Xi_{K}:=\left\{\xi \in \mathbb{R}^{K(\mathbb{C})} \mid \xi(\sigma)=\xi(\bar{\sigma})(\forall \sigma)\right\}, \\
\Xi_{K}^{0}:=\left\{\xi \in \Xi_{K} \mid \sum_{\sigma \in K(\mathbb{C})} \xi(\sigma)=0\right\} .
\end{array}\right.
$$

The Dirichlet unit theorem asserts that the group $O_{K}^{\times}$consisting of units in $O_{K}$ is a finitely generated abelian group of rank $s:=\operatorname{dim}_{\mathbb{R}} \Xi_{K}^{0}$.

Let us consider the homomorphism $L: K^{\times} \rightarrow \mathbb{R}^{K(\mathbb{C})}$ given by

$$
L(x)(\sigma):=\log |\sigma(x)| \quad\left(x \in K^{\times}, \sigma \in K(\mathbb{C})\right) .
$$

It is easy to see the following.

(a) For a compact set $B$ in $\mathbb{R}^{K(\mathbb{C})}$, the set $\left\{x \in O_{K}^{\times} \mid L(x) \in B\right\}$ is finite.

(b) $L: K^{\times} \rightarrow \mathbb{R}^{K(\mathbb{C})}$ extends to $L_{\mathbb{R}}: K^{\times} \otimes \mathbb{R} \rightarrow \mathbb{R}^{K(\mathbb{C})}$.

(c) $L_{\mathbb{R}}: O_{K}^{\times} \otimes \mathbb{R} \rightarrow \mathbb{R}^{K(\mathbb{C})}$ is injective.

(d) $L_{\mathbb{R}}\left(O_{K}^{\times} \otimes \mathbb{R}\right) \subseteq \Xi_{K}^{0}$.

By using (a) and (c), we can see that $O_{K}^{\times}$is a finitely generated abelian group. The most essential part of the Dirichlet unit theorem is to show that $O_{K}^{\times}$is of rank $s$, which is equivalent to seeing that, for any $\xi \in \Xi_{K}^{0}$, there is $x \in O_{K}^{\times} \otimes \mathbb{R}$ with $L_{\mathbb{R}}(x)=\xi$.

To understand the equality $L_{\mathbb{R}}(x)=\xi$ in terms of Arakelov geometry, let us introduce several notations for arithmetic divisors on the arithmetic curve $\operatorname{Spec}\left(O_{K}\right)$. An arithmetic $\mathbb{R}$-divisor on $\operatorname{Spec}\left(O_{K}\right)$ is a pair $(D, \xi)$ consisting of

Kyoto Journal of Mathematics, Vol. 55, No. 4 (2015), 799-817

DOI $10.1215 / 21562261-3157748$, (C) 2015 by Kyoto University

Received March 12, 2014. Revised August 18, 2014. Accepted September 18, 2014.

2010 Mathematics Subject Classification: Primary 14G15; Secondary 11G25, 11R04. 
an $\mathbb{R}$-divisor $D$ on $\operatorname{Spec}\left(O_{K}\right)$ and $\xi \in \Xi_{K}$. We often denote the pair $(D, \xi)$ by $\bar{D}$. The arithmetic principal $\mathbb{R}$-divisor $\widehat{(x)} \mathbb{R}_{\mathbb{R}}$ of $x \in K^{\times} \otimes \mathbb{R}$ is the arithmetic $\mathbb{R}$-divisor given by

$$
\widehat{(x)}_{\mathbb{R}}:=\left(\sum_{P} \operatorname{ord}_{P}(x)[P],-2 L_{\mathbb{R}}(x)\right),
$$

where $P$ runs over the set of all maximal ideals of $O_{K}$ and

$$
\operatorname{ord}_{P}(x):=a_{1} \operatorname{ord}_{P}\left(x_{1}\right)+\cdots+a_{r} \operatorname{ord}_{P}\left(x_{r}\right)
$$

for $x=x_{1}^{a_{1}} \cdots x_{r}^{a_{r}}\left(x_{1}, \ldots, x_{r} \in K^{\times}\right.$and $\left.a_{1}, \ldots, a_{r} \in \mathbb{R}\right)$. The arithmetic degree $\widehat{\operatorname{deg}}(\bar{D})$ of an arithmetic $\mathbb{R}$-divisor $\bar{D}=\left(\sum_{P} a_{P}[P], \xi\right)$ is defined to be

$$
\widehat{\operatorname{deg}}(\bar{D}):=\sum_{P} a_{P} \log \#\left(O_{K} / P\right)+\frac{1}{2} \sum_{\sigma \in K(\mathbb{C})} \xi(\sigma) .
$$

Note that

$$
\widehat{\operatorname{deg}}\left(\widehat{(x)}_{\mathbb{R}}\right)=0 \quad\left(x \in K^{\times} \otimes \mathbb{R}\right)
$$

by virtue of the product formula. Further, $\bar{D}=\left(\sum_{P} a_{P}[P], \xi\right)$ is said to be effective if $a_{P} \geq 0$ for all $P$ and $\xi(\sigma) \geq 0$ for all $\sigma$.

In [17, Section 3.4], we proved the following.

$$
\text { "If } \widehat{\operatorname{deg}}(\bar{D}) \geq 0 \text {, then } \bar{D}+\widehat{(x)})_{\mathbb{R}} \text { is effective for some } x \in K^{\times} \otimes \mathbb{R} \text {." }
$$

This implies the essential part of the Dirichlet unit theorem. Indeed, we set $\bar{D}=(0, \xi)$ for $\xi \in \Xi_{K}^{0}$. As $\widehat{\operatorname{deg}}(\bar{D})=0$, by $(0.1), \bar{D}+\widehat{(y)}_{\mathbb{R}}$ is effective for some $y \in K^{\times} \otimes \mathbb{R}$, and hence $\bar{D}+\widehat{(y)}_{\mathbb{R}}=(0,0)$ because $\widehat{\operatorname{deg}}\left(\bar{D}+\widehat{(y)}_{\mathbb{R}}\right)=0$. Here we set $y=u_{1}^{a_{1}} \cdots u_{r}^{a_{r}}$ such that $u_{1}, \ldots, u_{r} \in K^{\times}, a_{1}, \ldots, a_{r} \in \mathbb{R}$, and $a_{1}, \ldots, a_{r}$ are linearly independent over $\mathbb{Q}$. By using the linear independence of $a_{1}, \ldots, a_{r}$ over $\mathbb{Q}$, $\operatorname{ord}_{P}(y)=0$ implies that $\operatorname{ord}_{P}\left(u_{i}\right)=0$ for all maximal ideals $P$ of $O_{K}$ and $i=$ $1, \ldots, r$; that is, $u_{i} \in O_{K}^{\times}$for $i=1, \ldots, r$. Therefore, $\xi=L_{\mathbb{R}}\left(y^{2}\right)$ and $y \in O_{K}^{\times} \otimes \mathbb{R}$, as required. In this sense, (0.1) is an Arakelov-theoretic interpretation of the classical Dirichlet unit theorem.

In [17] and [18], we considered a higher-dimensional analogue of (0.1). In the higher-dimensional case, the condition " $\widehat{\operatorname{deg}}(\bar{D}) \geq 0$ " should be replaced by the pseudo-effectivity of $\bar{D}$. Of course, this analogue is not true in general (cf. [5]). It is, however, a very interesting problem to find a sufficient condition for the existence of an arithmetic small $\mathbb{R}$-section, that is, an element $x$ such that

$$
x=x_{1}^{a_{1}} \cdots x_{r}^{a_{r}} \quad\left(x_{1}, \ldots, x_{r} \text { are rational functions and } a_{1}, \ldots, a_{r} \in \mathbb{R}\right)
$$

and $\bar{D}+\widehat{(x)}_{\mathbb{R}}$ is effective. For example, in [17] and [18], we proved that if $D$ is numerically trivial and $\bar{D}$ is pseudo-effective, then $\bar{D}$ has an arithmetic small $\mathbb{R}$-section. In this article, we would like to consider a geometric analogue of the Dirichlet unit theorem in the above sense.

Let $X$ be a normal projective variety over an algebraically closed field $k$. Let $\operatorname{Div}(X)$ denote the group of Cartier divisors on $X$. Let $\mathbb{K}$ be either the field $\mathbb{Q}$ of rational numbers or the field $\mathbb{R}$ of real numbers. We define $\operatorname{Div}(X)_{\mathbb{K}}$ to be 
$\operatorname{Div}(X)_{\mathbb{K}}:=\operatorname{Div}(X) \otimes_{\mathbb{Z}} \mathbb{K}$, whose elements are called $\mathbb{K}$-Cartier divisors on $X$. For $\mathbb{K}$-Cartier divisors $D_{1}$ and $D_{2}$, we say that $D_{1}$ is $\mathbb{K}$-linearly equivalent to $D_{2}$, which is denoted by $D_{1} \sim_{\mathbb{K}} D_{2}$, if there are nonzero rational functions $\phi_{1}, \ldots, \phi_{r}$ on $X$ and $a_{1}, \ldots, a_{r} \in \mathbb{K}$ such that

$$
D_{1}-D_{2}=a_{1}\left(\phi_{1}\right)+\cdots+a_{r}\left(\phi_{r}\right) .
$$

Let $D$ be a $\mathbb{K}$-Cartier divisor on $X$. We say that $D$ is big if there is an ample $\mathbb{Q}$-Cartier divisor $A$ on $X$ such that $D-A$ is $\mathbb{K}$-linearly equivalent to an effective $\mathbb{K}$-Cartier divisor. Further, $D$ is said to be pseudo-effective if $D+B$ is big for any big $\mathbb{K}$-Cartier divisor $B$ on $X$. Note that if $D$ is $\mathbb{K}$-effective (i.e., $D$ is $\mathbb{K}$ linearly equivalent to an effective $\mathbb{K}$-Cartier divisor), then $D$ is pseudo-effective. The converse of the above statement holds on toric varieties (e.g., [4, Proposition 4.9]). However, it is not true in general. In the case where $k$ is uncountable (e.g., $k=\mathbb{C}$ ), several examples are known such as nontorsion numerically trivial invertible sheaves and Mumford's example on a minimal ruled surface (cf. [8, Chapter 1, Example 10.6], [14]). Nevertheless, we would like to propose the following question.

\section{QUESTION 0.2 (KK-VERSION)}

We assume that $k$ is an algebraic closure of a finite field. If a $\mathbb{K}$-Cartier divisor $D$ on $X$ is pseudo-effective, does it follow that $D$ is $\mathbb{K}$-effective?

This question is a geometric analogue of the fundamental question introduced in [17]. In this sense, it turns out to be a geometric Dirichlet's unit theorem if it is true, so that we often say that a $\mathbb{K}$-Cartier divisor $D$ has the Dirichlet property if $D$ is $\mathbb{K}$-effective. Note that the $\mathbb{R}$-version implies the $\mathbb{Q}$-version (cf. Proposition 1.5). Moreover, the $\mathbb{R}$-version does not hold in general. In Example 3.2, we give an example, so that, for the $\mathbb{R}$-version, the question should be

"Under what conditions does it follow that $D$ is $\mathbb{K}$-effective?"

Further, the $\mathbb{Q}$-version implies the following question due to Keel (cf. [10, Question 0.9], Remark 2.4). The similar arguments on an algebraic surface are discussed in the recent article by Langer [12, Conjectures 1.7-1.9 and Lemma 1.10].

\section{QUESTION 0.3 (S. KEEL)}

We assume that $k$ is an algebraic closure of a finite field and that $X$ is an algebraic surface over $k$. Let $D$ be a Cartier divisor on $X$. If $(D \cdot C)>0$ for all irreducible curves $C$ on $X$, is $D$ ample?

By virtue of the Zariski decomposition, Question 0.2 on an algebraic surface is equivalent to asking the following:

"If $D$ is nef, then is $D \mathbb{K}$-effective?" 
One might expect that $D$ is semiample (cf. [10, Question 0.8.2]). However, Totaro [23, Theorem 6.1] found a Cartier divisor $D$ on an algebraic surface over a finite field such that $D$ is nef but not semiample. Totaro's example does not give a counterexample to our question because we assert only the $\mathbb{Q}$-effectivity in Question 0.2. Inspired by Biswas and Subramanian [3], we have the following partial answer to the above question.

\section{THEOREM 0.4}

We assume that $k$ is an algebraic closure of a finite field. Let $C$ be a smooth projective curve over $k$, and let $E$ be a locally free sheaf of rank $r$ on $C$. Let $\mathbb{P}(E)$ be the projective bundle of $E$; that is, $\mathbb{P}(E):=\operatorname{Proj}\left(\bigoplus_{m=0}^{\infty} \operatorname{Sym}^{m}(E)\right)$. If $D$ is a pseudo-effective $\mathbb{K}$-Cartier divisor on $\mathbb{P}(E)$, then $D$ is $\mathbb{K}$-effective.

In addition to the above result, we can also give an affirmative answer to the $\mathbb{Q}$-version of Question 0.2 on abelian varieties.

\section{PROPOSITION 0.5}

We assume that $k$ is an algebraic closure of a finite field. Let $A$ be an abelian variety over $k$. If $D$ is a pseudo-effective $\mathbb{Q}$-Cartier divisor on $A$, then $D$ is $\mathbb{Q}$-effective.

\section{Preliminaries}

Let $k$ be an algebraic closed field. Let $C$ be a smooth projective curve over $k$, and let $E$ be a locally free sheaf of rank $r$ on $C$. The projective bundle $\mathbb{P}(E)$ of $E$ is given by

$$
\mathbb{P}(E):=\operatorname{Proj}\left(\bigoplus_{m=0}^{\infty} \operatorname{Sym}^{m}(E)\right) .
$$

The canonical morphism $\mathbb{P}(E) \rightarrow C$ is denoted by $f_{E}$. A tautological divisor $\Theta_{E}$ on $\mathbb{P}(E)$ is a Cartier divisor on $\mathbb{P}(E)$ such that $\mathcal{O}_{\mathbb{P}(E)}\left(\Theta_{E}\right)$ is isomorphic to the tautological invertible sheaf $\mathcal{O}_{\mathbb{P}(E)}(1)$ on $\mathbb{P}(E)$. We say that $E$ is strongly semistable if, for any surjective morphism $\pi: C^{\prime} \rightarrow C$ of smooth projective curves, $\pi^{*}(E)$ is semistable. By definition, if $E$ is strongly semistable and $\pi: C^{\prime} \rightarrow C$ is a surjective morphism of smooth projective curves over $k$, then $\pi^{*}(E)$ is also strongly semistable. A filtration

$$
0=E_{0} \subsetneq E_{1} \subsetneq E_{2} \subsetneq \cdots \subsetneq E_{s-1} \subsetneq E_{s}=E
$$

of $E$ is called the strong Harder-Narasimhan filtration if

$$
\mu\left(E_{1} / E_{0}\right)>\mu\left(E_{2} / E_{1}\right)>\cdots>\mu\left(E_{s-1} / E_{s-2}\right)>\mu\left(E_{s} / E_{s-1}\right)
$$

and $E_{i} / E_{i-1}$ is a strongly semistable locally free sheaf on $C$ for each $i=1, \ldots, s$. Recall the following well-known facts (F1)-(F5) on strong semistability. 
(F1) A locally free sheaf $E$ on $C$ is strong semistable if and only if $\Theta_{E}-$ $f_{E}^{*}\left(\xi_{E} / r\right)$ is nef, where $\xi_{E}$ is a Cartier divisor on $C$ with $\mathcal{O}_{C}\left(\xi_{E}\right) \simeq \operatorname{det}(E)$ (e.g., see [16, Proposition 7.1(3)]).

(F2) Let $\pi: C^{\prime} \rightarrow C$ be a surjective morphism of smooth projective curves over $k$ such that the function field of $C^{\prime}$ is a separable extension field over the function field of $C$. If $E$ is semistable, then $\pi^{*}(E)$ is also semistable (e.g., see [16, Proposition 7.1(1)]). In particular, if $\operatorname{char}(k)=0$, then $E$ is strongly semistable if and only if $E$ is semistable. Moreover, in the case where $\operatorname{char}(k)>0, E$ is strongly semistable if and only if $\left(F^{m}\right)^{*}(E)$ is semistable for all $m \geq 0$, where $F: C \rightarrow C$ is the absolute Frobenius map and

$$
F^{m}=\overbrace{F \circ \cdots \circ F}^{m} .
$$

(F3) If $E$ and $G$ are strongly semistable locally free sheaves on $C$, then $\operatorname{Sym}^{m}(E)$ and $E \otimes G$ are also strongly semistable for all $m \geq 1$ (e.g., see [16, Theorem 7.2, Corollary 7.3]).

(F4) There is a surjective morphism $\pi: C^{\prime} \rightarrow C$ of smooth projective curves over $k$ such that $\pi^{*}(E)$ has the strong Harder-Narasimhan filtration (cf. [11, Theorem 7.2]).

(F5) We assume that $k$ is an algebraic closure of a finite field. If $E$ is a strongly semistable locally free sheaf on $C$ with $\operatorname{det}(E) \simeq \mathcal{O}_{C}$, then there is a surjective morphism $\pi: C^{\prime} \rightarrow C$ of smooth projective curves over $k$ such that $\pi^{*}(E) \simeq \mathcal{O}_{C^{\prime}}^{\oplus \operatorname{rk} E}$ (cf. [1, p. 557], [22, Theorem 3.2], [3]).

The purpose of this section is to prove the following characterizations of pseudo-effective $\mathbb{R}$-Cartier divisors and nef $\mathbb{R}$-Cartier divisors on $\mathbb{P}(E)$. This result is essentially due to Nakayama [21, Lemma 3.7] in which he works over the complex number field.

\section{PROPOSITION 1.1}

We assume that $E$ has the strong Harder-Narasimhan filtration

$$
0=E_{0} \subsetneq E_{1} \subsetneq E_{2} \subsetneq \cdots \subsetneq E_{s-1} \subsetneq E_{s}=E .
$$

Then, for an $\mathbb{R}$-divisor $A$ on $C$, we have the following:

(a) $\Theta_{E}-f^{*}(A)$ is pseudo-effective if and only if $\operatorname{deg}(A) \leq \mu\left(E_{1}\right)$.

(b) $\Theta_{E}-f^{*}(A)$ is nef if and only if $\operatorname{deg}(A) \leq \mu\left(E / E_{s-1}\right)$.

Let us begin with the following lemma.

LEMMA 1.2

We assume that $E$ has a filtration

$$
0=E_{0} \subsetneq E_{1} \subsetneq \cdots \subsetneq E_{s-1} \subsetneq E_{s}=E
$$


such that $E_{i} / E_{i-1}$ is a strongly semistable locally free sheaf on $C$ and $\operatorname{deg}\left(E_{i} /\right.$ $\left.E_{i-1}\right)<0$ for all $i=1, \ldots, s$. Then, $H^{0}\left(C, \operatorname{Sym}^{m}(E) \otimes G\right)=0$ for $m \geq 1$ and $a$ strongly semistable locally free sheaf $G$ on $C$ with $\operatorname{deg}(G) \leq 0$.

Proof

We prove it by induction on $s$. In the case where $s=1, E$ is strongly semistable and $\operatorname{deg}(E)<0$, so that $\operatorname{Sym}^{m}(E) \otimes G$ is also strongly semistable by (F3) and

$$
\operatorname{deg}\left(\operatorname{Sym}^{m}(E) \otimes G\right)<0 .
$$

Therefore, $H^{0}\left(C, \operatorname{Sym}^{m}(E) \otimes G\right)=0$.

Here we assume that $s>1$. Let us consider an exact sequence

$$
0 \rightarrow E_{s-1} \rightarrow E \rightarrow E / E_{s-1} \rightarrow 0 .
$$

By [9, Chapter II, Exercise 5.16(c)], there is a filtration

$$
\operatorname{Sym}^{m}(E)=F^{0} \supsetneq F^{1} \supsetneq \cdots \supsetneq F^{m} \supsetneq F^{m+1}=0
$$

such that

$$
F^{j} / F^{j+1} \simeq \operatorname{Sym}^{j}\left(E_{s-1}\right) \otimes \operatorname{Sym}^{m-j}\left(E / E_{s-1}\right)
$$

for each $j=0, \ldots, m$. By using the hypothesis of induction,

$$
H^{0}\left(C,\left(F^{j} / F^{j+1}\right) \otimes G\right)=0
$$

for $j=1, \ldots, m$ because $\operatorname{Sym}^{m-j}\left(E / E_{s-1}\right) \otimes G$ is strongly semistable by (F3) and

$$
\operatorname{deg}\left(\operatorname{Sym}^{m-j}\left(E / E_{s-1}\right) \otimes G\right) \leq 0 .
$$

Moreover, since $\operatorname{Sym}^{m}\left(E / E_{s-1}\right) \otimes G$ is strongly semistable by (F3) and

$$
\operatorname{deg}\left(\operatorname{Sym}^{m}\left(E / E_{s-1}\right) \otimes G\right)<0,
$$

we have that

$$
H^{0}\left(C,\left(F^{0} / F^{1}\right) \otimes G\right)=H^{0}\left(C, \operatorname{Sym}^{m}\left(E / E_{s-1}\right) \otimes G\right)=0 .
$$

Therefore, by using an exact sequence

$$
0 \rightarrow F^{j+1} \otimes G \rightarrow F^{j} \otimes G \rightarrow\left(F^{j} / F^{j+1}\right) \otimes G \rightarrow 0,
$$

we have that

$$
H^{0}\left(C, F^{j+1} \otimes G\right) \stackrel{\sim}{\longrightarrow} H^{0}\left(C, F^{j} \otimes G\right)
$$

for $j=0, \ldots, m$, which implies that $H^{0}\left(C, \operatorname{Sym}^{m}(E) \otimes G\right)=0$, as required.

Proof of Proposition 1.1

It is sufficient to show the following.

(a) If $A$ is a $\mathbb{Q}$-Cartier divisor and $\operatorname{deg}(A)<\mu\left(E_{1}\right)$, then $\Theta_{E}-f^{*}(A)$ is Q-effective. 
(b) If $A$ is a $\mathbb{Q}$-Cartier divisor and $\operatorname{deg}(A)>\mu\left(E_{1}\right)$, then $\Theta_{E}-f^{*}(A)$ is not pseudo-effective.

(c) If $\Theta_{E}-f^{*}(A)$ is nef, then $\operatorname{deg}(A) \leq \mu\left(E / E_{s-1}\right)$.

(d) If $\Theta_{E}-f^{*}(A)$ is not nef, then $\operatorname{deg}(A)>\mu\left(E / E_{s-1}\right)$.

(a) Let $\theta$ be a divisor on $C$ with $\operatorname{deg}(\theta)=1$. As $E_{1}$ is strongly semistable, by (F1), $\Theta_{E_{1}}-\mu\left(E_{1}\right) f_{E_{1}}^{*}(\theta)$ is nef, so that we can see that $\Theta_{E_{1}}-f_{E_{1}}^{*}(A)$ is nef and big because

$$
\Theta_{E_{1}}-\operatorname{deg}(A) f_{E_{1}}^{*}(\theta)=\Theta_{E_{1}}-\mu\left(E_{1}\right) f_{E_{1}}^{*}(\theta)+\left(\mu\left(E_{1}\right)-\operatorname{deg}(A)\right) f_{E_{1}}^{*}(\theta) .
$$

Therefore, there is a positive integer $m_{1}$ such that $m_{1} A$ is a divisor on $C$ and

$$
H^{0}\left(\mathbb{P}\left(E_{1}\right), \mathcal{O}_{\mathbb{P}\left(E_{1}\right)}\left(m_{1} \Theta_{E_{1}}-f_{E_{1}}^{*}\left(m_{1} A\right)\right)\right) \neq 0
$$

In addition,

$$
\begin{aligned}
H^{0} & \left(\mathbb{P}\left(E_{1}\right), \mathcal{O}_{\mathbb{P}\left(E_{1}\right)}\left(m_{1} \Theta_{E_{1}}-f_{E_{1}}^{*}\left(m_{1} A\right)\right)\right) \\
& =H^{0}\left(C, \operatorname{Sym}^{m_{1}}\left(E_{1}\right) \otimes \mathcal{O}_{C}\left(-m_{1} A\right)\right) \\
& \subseteq H^{0}\left(C, \operatorname{Sym}^{m_{1}}(E) \otimes \mathcal{O}_{C}\left(-m_{1} A\right)\right) \\
& =H^{0}\left(\mathbb{P}(E), \mathcal{O}_{\mathbb{P}(E)}\left(m_{1} \Theta_{E}-f_{E}^{*}\left(m_{1} A\right)\right)\right),
\end{aligned}
$$

so that $\Theta_{E}-f_{E}^{*}(A)$ is $\mathbb{Q}$-effective.

(b) Let $B$ be an ample $\mathbb{Q}$-divisor on $C$ with $\operatorname{deg}(B)<\operatorname{deg}(A)-\mu\left(E_{1}\right)$. Let $\pi: C^{\prime} \rightarrow C$ be a surjective morphism of smooth projective curves over $k$ such that $\pi^{*}(-A+B)$ is a Cartier divisor on $C^{\prime}$. Note that

$$
\mu\left(\pi^{*}\left(E_{i} / E_{i-1}\right) \otimes \mathcal{O}_{C^{\prime}}\left(\pi^{*}(-A+B)\right)\right)<0
$$

for $i=1, \ldots, s$, and hence, by Lemma 1.2,

$$
H^{0}\left(C^{\prime}, \operatorname{Sym}^{m}\left(\pi^{*}(E)\right) \otimes \mathcal{O}_{C^{\prime}}\left(m \pi^{*}(-A+B)\right)\right)=0
$$

for all $m \geq 1$. In particular, if $b$ is a positive integer such that $b(-A+B)$ is a Cartier divisor, then

$$
H^{0}\left(C, \operatorname{Sym}^{m b}(E) \otimes \mathcal{O}_{C}(m b(-A+B))\right)=0
$$

for $m \geq 1$. Here we assume that $\Theta_{E}-f_{E}^{*}(A)$ is pseudo-effective. Let $a$ be a positive integer such that $\Theta_{E}-f_{E}^{*}(A)+a f_{E}^{*}(B)$ is ample. Then

$$
(a-1)\left(\Theta_{E}-f_{E}^{*}(A)\right)+\Theta_{E}-f_{E}^{*}(A)+a f_{E}^{*}(B)=a\left(\Theta_{E}+f_{E}^{*}(-A+B)\right)
$$

is big, so that we can find a positive integer $m_{1}$ such that

$$
\begin{aligned}
& H^{0}\left(C, \operatorname{Sym}^{m_{1} a b}(E) \otimes \mathcal{O}_{C}\left(m_{1} a b(-A+B)\right)\right) \\
& \quad=H^{0}\left(\mathbb{P}(E), \mathcal{O}_{\mathbb{P}(E)}\left(m_{1} a b\left(\Theta_{E}+f_{E}^{*}(-A+B)\right)\right)\right) \neq 0,
\end{aligned}
$$

which is a contradiction. 
(c) Note that

$$
\begin{aligned}
\mathbb{P}\left(E / E_{s-1}\right) & \subseteq \mathbb{P}(E),\left.\quad \Theta_{E / E_{s-1}} \sim \Theta_{E}\right|_{\mathbb{P}\left(E / E_{s-1}\right)}, \quad \text { and } \\
f_{E / E_{s-1}} & =\left.f_{E}\right|_{\mathbb{P}\left(E / E_{s-1}\right)}
\end{aligned}
$$

so that $\Theta_{E / E_{s-1}}-f_{E / E_{s-1}}^{*}(A)$ is nef on $\mathbb{P}\left(E / E_{s-1}\right)$. Let $\xi_{E / E_{s-1}}$ be a Cartier divisor on $C$ with $\mathcal{O}_{C}\left(\xi_{E / E_{s-1}}\right) \simeq \operatorname{det}\left(E / E_{s-1}\right)$. If we set $e=\operatorname{rk} E / E_{s-1}$ and $G=\xi_{E / E_{s-1}} / e-A$, then

$$
\Theta_{E / E_{s-1}}-f_{E / E_{s-1}}^{*}(A)=\Theta_{E / E_{s-1}}-f_{E / E_{s-1}}^{*}\left(\xi_{E / E_{s-1}} / e\right)+f_{E / E_{s-1}}^{*}(G) .
$$

Since $\Theta_{E / E_{s-1}}-f_{E / E_{s-1}}^{*}\left(\xi_{E / E_{s-1}} / e\right)$ is nef by (F1) and

$$
\left(\Theta_{E / E_{s-1}}-f_{E / E_{s-1}}^{*}\left(\xi_{E / E_{s-1}} / e\right)\right)^{e}=0
$$

we have that

$$
0 \leq\left(\Theta_{E / E_{s-1}}-f_{E / E_{s-1}}^{*}(A)\right)^{e}=e \operatorname{deg}(G) .
$$

Therefore, $\operatorname{deg}(G) \geq 0$, and hence $\operatorname{deg}(A) \leq \mu\left(E / E_{s-1}\right)$.

(d) We can find an irreducible curve $C_{0}$ of $X$ such that $\left(\Theta_{E}-f_{E}^{*}(A) \cdot C_{0}\right)<0$. Clearly $C_{0}$ is flat over $C$. Let $C_{1}$ be the normalization of $C_{0}$, and let $h: C_{1} \rightarrow$ $C$ be the induced morphism. Let us consider the following commutative diagram:

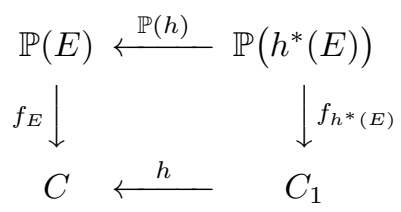

Note that $\mathbb{P}(h)^{*}\left(\Theta_{E}-f_{E}^{*}(A)\right) \sim_{\mathbb{R}} \Theta_{h^{*}(E)}-f_{h^{*}(E)}^{*}\left(h^{*}(A)\right)$. Further, there is a section $S$ of $f_{h^{*}(E)}$ such that $\mathbb{P}(h)_{*}(S)=C_{0}$. Let $Q$ be the quotient line bundle of $h^{*}(E)$ corresponding to the section $S$. As

$$
0=h^{*}\left(E_{0}\right) \subsetneq h^{*}\left(E_{1}\right) \subsetneq h^{*}\left(E_{2}\right) \subsetneq \cdots \subsetneq h^{*}\left(E_{s-1}\right) \subsetneq h^{*}\left(E_{s}\right)=h^{*}(E)
$$

is the Harder-Narasimhan filtration of $h^{*}(E)$, we can easily see that

$$
\operatorname{deg}(Q) \geq \mu\left(h^{*}\left(E / E_{s-1}\right)\right)=\operatorname{deg}(h) \mu\left(E / E_{s-1}\right) .
$$

On the other hand,

$$
\begin{aligned}
\operatorname{deg}(Q)-\operatorname{deg}(h) \operatorname{deg}(A) & =\left(\Theta_{h^{*}(E)}-f_{h^{*}(E)}^{*}\left(h^{*}(A)\right) \cdot S\right) \\
& =\left(\Theta_{E}-f_{E}^{*}(A) \cdot C_{0}\right)<0,
\end{aligned}
$$

and hence $\mu\left(E / E_{s-1}\right)<\operatorname{deg}(A)$.

Finally let us consider the following three results.

\section{LEMMA 1.3}

Let $\mathbb{K}$ be either $\mathbb{Q}$ or $\mathbb{R}$. Let $\mu: X^{\prime} \rightarrow X$ be a generically finite morphism of normal 
projective varieties over $k$. For a $\mathbb{K}$-Cartier divisor $D$ on $X, D$ is $\mathbb{K}$-effective if and only if $\mu^{*}(D)$ is $\mathbb{K}$-effective.

Proof

Clearly, if $D$ is $\mathbb{K}$-effective, then $\mu^{*}(D)$ is $\mathbb{K}$-effective. Let $K$ and $K^{\prime}$ be the function fields of $X$ and $X^{\prime}$, respectively. Here we assume that $\mu^{*}(D)$ is $\mathbb{K}$ effective; that is, there are $\phi_{1}^{\prime}, \ldots, \phi_{r}^{\prime} \in K^{\prime \times}$ and $a_{1}, \ldots, a_{r} \in \mathbb{K}$ such that $\mu^{*}(D)+$ $a_{1}\left(\phi_{1}^{\prime}\right)+\cdots+a_{r}\left(\phi_{r}^{\prime}\right)$ is effective, so that

$$
\mu_{*}\left(\mu^{*}(D)+a_{1}\left(\phi_{1}^{\prime}\right)+\cdots+a_{r}\left(\phi_{r}^{\prime}\right)\right)=\operatorname{deg}(\mu) D+a_{1} \mu_{*}\left(\left(\phi_{1}^{\prime}\right)\right)+\cdots+a_{r} \mu_{*}\left(\left(\phi_{r}^{\prime}\right)\right)
$$

is effective. Note that $\mu_{*}\left(\phi_{i}^{\prime}\right)=\left(N_{K^{\prime} / K}\left(\phi_{i}^{\prime}\right)\right)$ (cf. [7, Proposition 1.4]), where $N_{K^{\prime} / K}$ is the norm map of $K^{\prime}$ over $K$, and hence

$$
D+\left(a_{1} / \operatorname{deg}(\mu)\right)\left(N_{K^{\prime} / K}\left(\phi_{1}^{\prime}\right)\right)+\cdots+\left(a_{r} / \operatorname{deg}(\mu)\right)\left(N_{K^{\prime} / K}\left(\phi_{r}^{\prime}\right)\right)
$$

is effective. Therefore, $D$ is $\mathbb{K}$-effective.

\section{LEMMA 1.4}

Let $\mathbb{K}$ be either $\mathbb{Q}$ or $\mathbb{R}$. We assume that $k$ is an algebraic closure of a finite field. Let $X$ be a normal projective variety over $k$, and let $D$ be a $\mathbb{K}$-Cartier divisor on $X$. If $D$ is numerically trivial, then $D$ is $\mathbb{K}$-linearly equivalent to the zero divisor.

Proof

If $\mathbb{K}=\mathbb{Q}$, then the assertion is well known, so that we assume that $\mathbb{K}=\mathbb{R}$. We set $D=a_{1} D_{1}+\cdots+a_{r} D_{r}$, where $D_{1}, \ldots, D_{r}$ are Cartier divisors on $X$ and $a_{1}, \ldots, a_{r} \in \mathbb{R}$. Considering a $\mathbb{Q}$-basis of $\mathbb{Q} a_{1}+\cdots+\mathbb{Q} a_{r}$ in $\mathbb{R}$, we may assume that $a_{1}, \ldots, a_{r}$ are linearly independent over $\mathbb{Q}$. Let $C$ be an irreducible curve on $X$. Note that

$$
0=(D \cdot C)=a_{1}\left(D_{1} \cdot C\right)+\cdots+a_{r}\left(D_{r} \cdot C\right)
$$

and $\left(D_{1} \cdot C\right), \ldots,\left(D_{r} \cdot C\right) \in \mathbb{Z}$, and hence $\left(D_{1} \cdot C\right)=\cdots=\left(D_{r} \cdot C\right)=0$ because $a_{1}, \ldots, a_{r}$ are linearly independent over $\mathbb{Q}$. Thus, $D_{1}, \ldots, D_{r}$ are numerically equivalent to zero, so that $D_{1}, \ldots, D_{r}$ are $\mathbb{Q}$-linearly equivalent to the zero divisor. Therefore, the assertion follows.

PROPOSITION 1.5

Let $X$ be a normal projective variety over $k$, and let $D$ be a $\mathbb{Q}$-Cartier divisor on $X$. If $D$ is $\mathbb{R}$-effective, then $D$ is $\mathbb{Q}$-effective.

\section{Proof}

As $D$ is $\mathbb{R}$-effective, there are nonzero rational functions $\psi_{1}, \ldots, \psi_{l}$ on $X$ and $b_{1}, \ldots, b_{l} \in \mathbb{R}$ such that $D+b_{1}\left(\psi_{1}\right)+\cdots+b_{l}\left(\psi_{l}\right)$ is effective. We set $V=\mathbb{Q} b_{1}+$ $\cdots+\mathbb{Q} b_{l} \subseteq \mathbb{R}$. If $V \subseteq \mathbb{Q}$, then $b_{1}, \ldots, b_{l} \in \mathbb{Q}$, so that we may assume that $V \nsubseteq \mathbb{Q}$. 
CLAIM 1.5.1

There are nonzero rational functions $\phi_{1}, \ldots, \phi_{r}$ on $X, a_{1}, \ldots, a_{r} \in \mathbb{R}$, and $a \mathbb{Q}$ Cartier divisor $D^{\prime}$ on $X$ such that $D \sim_{\mathbb{Q}} D^{\prime}, D^{\prime}+a_{1}\left(\phi_{1}\right)+\cdots+a_{r}\left(\phi_{r}\right)$ is effective, and $1, a_{1}, \ldots, a_{r}$ are linearly independent over $\mathbb{Q}$.

Proof

We can find a basis $a_{1}, \ldots, a_{r}$ of $V$ over $\mathbb{Q}$ with the following properties:

(i) If we set $b_{i}=\sum_{j=1}^{r} c_{i j} a_{j}$, then $c_{i j} \in \mathbb{Z}$ for all $i, j$.

(ii) If $V \cap \mathbb{Q} \neq\{0\}$, then $a_{1} \in \mathbb{Q}^{\times}$.

We put $\phi_{j}=\prod_{i=1}^{l} \psi_{i}^{c_{i j}}$. Note that $\sum_{i=1}^{l} b_{i}\left(\psi_{i}\right)=\sum_{j=1}^{r} a_{j}\left(\phi_{j}\right)$. Therefore, in the case where $V \cap \mathbb{Q}=\{0\}, 1, a_{1}, \ldots, a_{r}$ are linearly independent over $\mathbb{Q}$ and $D+$ $\sum_{j=1}^{r} a_{j}\left(\phi_{j}\right)$ is effective. Otherwise, $1, a_{2}, \ldots, a_{r}$ are linearly independent over $\mathbb{Q}$ and $\left(D+a_{1}\left(\phi_{1}\right)\right)+\sum_{j=2}^{r} a_{j}\left(\phi_{j}\right)$ is effective.

We set $L=D^{\prime}+a_{1}\left(\phi_{1}\right)+\cdots+a_{r}\left(\phi_{r}\right)$. Let $\Gamma$ be a prime divisor with $\Gamma \nsubseteq \operatorname{Supp}(L)$. Then

$$
0=\operatorname{mult}_{\Gamma}(L)=\operatorname{mult}_{\Gamma}\left(D^{\prime}\right)+a_{1} \operatorname{ord}_{\Gamma}\left(\phi_{1}\right)+\cdots+a_{r} \operatorname{ord}_{\Gamma}\left(\phi_{r}\right),
$$

so that $\operatorname{mult}_{\Gamma}\left(D^{\prime}\right)=\operatorname{ord}_{\Gamma}\left(\phi_{1}\right)=\cdots=\operatorname{ord}_{\Gamma}\left(\phi_{r}\right)=0$ because $1, a_{1}, \ldots, a_{r}$ are linearly independent over $\mathbb{Q}$. Thus,

$$
\operatorname{Supp}\left(D^{\prime}\right), \operatorname{Supp}\left(\left(\phi_{1}\right)\right), \ldots, \operatorname{Supp}\left(\left(\phi_{r}\right)\right) \subseteq \operatorname{Supp}(L) .
$$

Therefore, we can find $a_{1}^{\prime}, \ldots, a_{r}^{\prime} \in \mathbb{Q}$ such that $D^{\prime}+a_{1}^{\prime}\left(\phi_{1}\right)+\cdots+a_{r}^{\prime}\left(\phi_{r}\right)$ is effective, and hence $D$ is $\mathbb{Q}$-effective.

\section{Proof of Theorem 0.4}

Let $k$ be an algebraic closure of a finite field. Let $C$ be a smooth projective curve over $k$. Let us begin with the following lemma.

\section{LEMMA 2.1}

Let $\mathbb{K}$ be either $\mathbb{Q}$ or $\mathbb{R}$. Let $A$ be a $\mathbb{K}$-Cartier divisor on $C$. If $\operatorname{deg}(A) \geq 0$, then $A$ is $\mathbb{K}$-effective.

Proof

If $\mathbb{K}=\mathbb{Q}$, then the assertion is obvious. We assume that $\mathbb{K}=\mathbb{R}$. If $\operatorname{deg}(A)=0$, then the assertion follows from Lemma 1.4. Next we consider the case where $\operatorname{deg}(A)>0$. We can find a $\mathbb{Q}$-Cartier divisor $A^{\prime}$ such that $A^{\prime} \leq A$ and $\operatorname{deg}\left(A^{\prime}\right)>0$. Thus, the previous observation implies the assertion.

As a consequence of (F3), (F4), and (F5), we have the following splitting theorem, which was obtained by Biswas and Parameswaran [2, Proposition 2.1]. 


\section{THEOREM 2.2}

For a locally free sheaf $E$ on $C$, there are a surjective morphism $\pi: C^{\prime} \rightarrow C$ of smooth projective curves over $k$ and invertible sheaves $L_{1}, \ldots, L_{r}$ on $C^{\prime}$ such that $\pi^{*}(E) \simeq L_{1} \oplus \cdots \oplus L_{r}$.

\section{Proof}

For the reader's convenience, we give a sketch of the proof. First we assume that $E$ is strongly semistable. Let $\xi_{E}$ be a Cartier divisor on $C$ with $\mathcal{O}_{C}\left(\xi_{E}\right) \simeq \operatorname{det}(E)$. Let $h: B \rightarrow C$ be a surjective morphism of smooth projective curves over $k$ such that $h^{*}\left(\xi_{E}\right)$ is divisible by $\operatorname{rk}(E)$. We set $E^{\prime}=h^{*}(E) \otimes \mathcal{O}_{B}\left(-h^{*}\left(\xi_{E}\right) / \operatorname{rk}(E)\right)$. As $\operatorname{det}\left(E^{\prime}\right) \simeq \mathcal{O}_{B}$, the assertion follows from (F5).

By the above observation, it is sufficient to find a surjective morphism $\pi$ : $C^{\prime} \rightarrow C$ of smooth projective curves over $k$ and strongly semistable locally free sheaves $Q_{1}, \ldots, Q_{n}$ on $C^{\prime}$ such that

$$
\pi^{*}(E)=Q_{1} \oplus \cdots \oplus Q_{n} .
$$

Moreover, by (F4), we may assume that $E$ has the strong Harder-Narasimhan filtration

$$
0=E_{0} \subsetneq E_{1} \subsetneq E_{2} \subsetneq \cdots \subsetneq E_{n-1} \subsetneq E_{n}=E .
$$

Clearly we may further assume that $n \geq 2$. For a nonnegative integer $m$, we set

$$
C_{m}:=X \times_{\operatorname{Spec}(k)} \operatorname{Spec}(k),
$$

where the morphism $\operatorname{Spec}(k) \rightarrow \operatorname{Spec}(k)$ is given by $x \mapsto x^{1 / p^{m}}$. Let $F_{k}^{m}: C_{m} \rightarrow C$ be the relative $m$ th Frobenius morphism over $k$. Put

$$
G_{i, j}^{m}:=\left(F_{k}^{m}\right)^{*}\left(\left(E_{j} / E_{i}\right) \otimes\left(E_{i} / E_{i-1}\right)^{\vee}\right) \otimes \omega_{C_{m}}
$$

for $i=1, \ldots, n-1$ and $j=i, \ldots, n$. We can find a positive integer $m$ such that

$$
\mu\left(G_{i, i+1}^{m}\right)=p^{m}\left(\mu\left(E_{i+1} / E_{i}\right)-\mu\left(E_{i} / E_{i-1}\right)\right)+\operatorname{deg}\left(\omega_{C}\right)<0
$$

for all $i=1, \ldots, n-1$. By using (F3), we can see that

$$
0=G_{i, i}^{m} \subsetneq G_{i, i+1}^{m} \subsetneq G_{i, i+2}^{m} \subsetneq \cdots \subsetneq G_{i, n-1}^{m} \subsetneq G_{i, n}^{m}
$$

is the strong Harder-Narasimhan filtration of $G_{i, n}^{m}$, so that $H^{0}\left(C_{m}, G_{i, n}^{m}\right)=\{0\}$, which yields

$$
\operatorname{Ext}^{1}\left(\left(F_{k}^{m}\right)^{*}\left(E / E_{i}\right),\left(F_{k}^{m}\right)^{*}\left(E_{i} / E_{i-1}\right)\right)=0
$$

because of Serre's duality theorem. Therefore, an exact sequence

$$
0 \rightarrow\left(F_{k}^{m}\right)^{*}\left(E_{i} / E_{i-1}\right) \rightarrow\left(F_{k}^{m}\right)^{*}\left(E / E_{i-1}\right) \rightarrow\left(F_{k}^{m}\right)^{*}\left(E / E_{i}\right) \rightarrow 0
$$

splits; that is, $\left(F_{k}^{m}\right)^{*}\left(E / E_{i-1}\right) \simeq\left(F_{k}^{m}\right)^{*}\left(E_{i} / E_{i-1}\right) \oplus\left(F_{k}^{m}\right)^{*}\left(E / E_{i}\right)$ for $i=1, \ldots$, $n-1$, and hence

$$
\left(F_{k}^{m}\right)^{*}(E) \simeq \bigoplus_{i=1}^{n}\left(F_{k}^{m}\right)^{*}\left(E_{i} / E_{i-1}\right)
$$

as required. 
Proof of Theorem 0.4

By virtue of Theorem 2.2 and Lemma 1.3, we may assume that

$$
E \simeq L_{1} \oplus \cdots \oplus L_{r}
$$

for some invertible sheaves $L_{1}, \ldots, L_{r}$ on $C$. We set

$$
d=\max \left\{\operatorname{deg}\left(L_{1}\right), \ldots, \operatorname{deg}\left(L_{r}\right)\right\} \quad \text { and } \quad I=\left\{i \mid \operatorname{deg}\left(L_{i}\right)=d\right\} .
$$

There is a $\mathbb{K}$-Cartier divisor $A$ on $C$ such that $D \sim_{\mathbb{K}} \lambda \Theta_{E}-f_{E}^{*}(A)$ for some $\lambda \in \mathbb{K}$. Let $M$ be an ample divisor on $C$ such that $T:=\Theta_{E}+f_{E}^{*}(M)$ is ample. As $D$ is pseudo-effective, we have that

$$
0 \leq\left(D \cdot T^{r-2} \cdot f_{E}^{*}(M)\right)=\left(\left(\lambda T-f_{E}^{*}(A+\lambda M)\right) \cdot T^{r-2} \cdot f_{E}^{*}(M)\right)=\lambda \operatorname{deg}(M),
$$

and hence $\lambda \geq 0$. If $\lambda=0$, then $0 \leq\left(D \cdot T^{r-1}\right)=\operatorname{deg}(-A)$. Thus, by Lemma 2.1, $-A$ is $\mathbb{K}$-effective, so that the assertion follows.

We assume that $\lambda>0$. Replacing $D$ by $D / \lambda$, we may assume that $\lambda=1$. Let $\xi$ be a Cartier divisor on $C$ such that $\mathcal{O}_{C}(\xi) \simeq L_{i_{0}}$ for some $i_{0} \in I$. Note that the first part $E_{1}$ of the strong Harder-Narasimhan filtration of $E$ is $\bigoplus_{i \in I} L_{i}$, so that, by Proposition $1.1, \operatorname{deg}(A) \leq \operatorname{deg}(\xi)$. If we set $B=\xi-A$, then, by Lemma 2.1, $B$ is $\mathbb{K}$-effective because $\operatorname{deg}(B) \geq 0$. Moreover, as

$$
\Theta_{E}-f_{E}^{*}(A)=\Theta_{E}-f_{E}^{*}(\xi)+f_{E}^{*}(B)
$$

it is sufficient to consider the case where $D=\Theta_{E}-f_{E}^{*}(\xi)$. In this case, the assertion is obvious because

$$
\begin{aligned}
H^{0}\left(\mathbb{P}(E), \mathcal{O}_{\mathbb{P}(E)}(D)\right) & =H^{0}\left(C, E \otimes \mathcal{O}_{C}(-\xi)\right) \\
& =H^{0}\left(C, \bigoplus_{i=1}^{r} L_{i} \otimes \mathcal{O}_{C}(-\xi)\right) \neq\{0\}
\end{aligned}
$$

As a consequence of Theorem 0.4 , we can recover a result due to [3].

\section{COROLLARY 2.3}

Let $k, C$, and $E$ be the same as in Theorem 0.4. We assume that $r=2$. Let $D$ be a Cartier divisor on $\mathbb{P}(E)$ such that $(D \cdot Y)>0$ for all irreducible curves $Y$ on $\mathbb{P}(E)$. Then $D$ is ample.

\section{Proof}

As $D$ is nef, $D$ is pseudo-effective, so that, by Theorem 0.4 , there is an effective $\mathbb{Q}$-Cartier divisor $E$ on $X$ such that $D \sim_{\mathbb{Q}} E$. As $E \neq 0$, we have that $(D \cdot D)=$ $(D \cdot E)>0$. Therefore, $D$ is ample by the Nakai-Moishezon criterion.

\section{REMARK 2.4}

The argument in the proof of Corollary 2.3 actually shows that the $\mathbb{Q}$-version of Question 0.2 on algebraic surfaces implies Question 0.3. 


\section{Numerical effectivity on abelian varieties}

The purpose of this section is to give an affirmative answer for the $\mathbb{Q}$-version of Question 0.2 on abelian varieties. Let $A$ be an abelian variety over an algebraically closed field $k$. A key observation is the following proposition.

PROPOSITION 3.1

If a $\mathbb{Q}$-Cartier divisor $D$ on $A$ is nef, then $D$ is numerically equivalent to a $\mathbb{Q}$-effective $\mathbb{Q}$-Cartier divisor.

\section{Proof}

We prove it by induction on $\operatorname{dim} A$. If $\operatorname{dim} A \leq 1$, then the assertion is obvious. Clearly we may assume that $D$ is a Cartier divisor, so that we set $L=\mathcal{O}_{A}(D)$. As $L \otimes[-1]^{*}(L)$ is numerically equivalent to $L^{\otimes 2}$ (cf. [20, p. 75 , (iv)]), we may assume that $L$ is symmetric; that is, $L \simeq[-1]^{*}(L)$. Let $K(L)$ be the closed subgroup of $A$ given by $K(L)=\left\{x \in A \mid T_{x}^{*}(L) \simeq L\right\}$ (cf. [20, p. 60, Definition]). If $K(L)$ is finite, then $L$ is nef and big by virtue of [20, p. 150, Riemann-Roch theorem], so that $D$ is $\mathbb{Q}$-effective. Otherwise, let $B$ be the connected component of $K(L)$ containing 0 .

\section{CLAIM 3.1.1}

(a) $\left.\left.T_{x}^{*}(L)\right|_{B} \simeq L\right|_{B}$ for all $x \in A$.

(b) $\left.L^{\otimes 2}\right|_{B+x} \simeq \mathcal{O}_{B+x}$ for $x \in A$.

Proof

(a) Let $N$ be an invertible sheaf on $A \times A$ given by

$$
N=m^{*}(L) \otimes p_{1}^{*}\left(L^{-1}\right) \otimes p_{2}^{*}\left(L^{-1}\right),
$$

where $p_{i}: A \times A \rightarrow A$ is the projection to the $i$ th factor $(i=1,2)$ and $m$ is the addition morphism. Note that $\left.N\right|_{B \times A} \simeq \mathcal{O}_{B \times A}$ (cf. [20, Section 13, p. 123]). Fixing $x \in A$, let us consider a morphism $\alpha: B \rightarrow B \times A$ given by $\alpha(y)=(y, x)$. Then

$$
\left.\left.\mathcal{O}_{B} \simeq \alpha^{*}\left(\left.m^{*}(L) \otimes p_{1}^{*}\left(L^{-1}\right) \otimes p_{2}^{*}\left(L^{-1}\right)\right|_{B \times A}\right) \simeq T_{x}^{*}(L)\right|_{B} \otimes L^{-1}\right|_{B},
$$

as required.

(b) First we consider the case where $x=0$. As $\left.N\right|_{B \times A} \simeq \mathcal{O}_{B \times A}$, we have that $\left.N\right|_{B \times B} \simeq \mathcal{O}_{B \times B}$. Using a morphism $\beta: B \rightarrow B \times B$ given by $\beta(y)=(y,-y)$, we have that

$$
\mathcal{O}_{B} \simeq \beta^{*}\left(\left.N\right|_{B \times B}\right)=\left.\left.\left.L^{-1}\right|_{B} \otimes[-1]^{*}\left(L^{-1}\right)\right|_{B} \simeq L^{\otimes-2}\right|_{B},
$$

as required. 
In general, for $x \in A$, by (a) and the previous observation together with the following commutative diagram

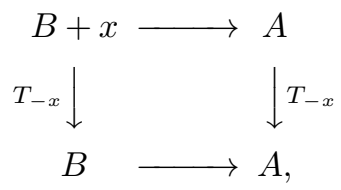

we can see that

$$
\begin{aligned}
\mathcal{O}_{B+x} & =T_{-x}^{*}\left(\mathcal{O}_{B}\right) \simeq T_{-x}^{*}\left(\left.L^{\otimes 2}\right|_{B}\right) \simeq T_{-x}^{*}\left(\left.T_{x}^{*}(L)^{\otimes 2}\right|_{B}\right) \\
& =T_{-x}^{*}\left(\left.T_{x}^{*}\left(L^{\otimes 2}\right)\right|_{B}\right)=\left.T_{-x}^{*}\left(T_{x}^{*}\left(L^{\otimes 2}\right)\right)\right|_{B+x}=\left.L^{\otimes 2}\right|_{B+x} .
\end{aligned}
$$

Let $\pi: A \rightarrow A / B$ be the canonical homomorphism. By Claim 3.1.1(b),

$$
\operatorname{dim}_{k(y)} H^{0}\left(\pi^{-1}(y), L^{\otimes 2}\right)=1
$$

for all $y \in A / B$, so that, by [20, p. 51, Corollary 2], $\pi_{*}\left(L^{\otimes 2}\right)$ is an invertible sheaf on $A / B$ and $\pi_{*}\left(L^{\otimes 2}\right) \otimes k(y) \stackrel{\sim}{\longrightarrow} H^{0}\left(\pi^{-1}(y), L^{\otimes 2}\right)$. Therefore, the natural homomorphism $\pi^{*}\left(\pi_{*}\left(L^{\otimes 2}\right)\right) \rightarrow L^{\otimes 2}$ is an isomorphism; that is, there is a $\mathbb{Q}$ Cartier divisor $D^{\prime}$ on $A / B$ such that $\pi^{*}\left(D^{\prime}\right) \sim_{\mathbb{Q}} D$. Note that $D^{\prime}$ is also nef, so that, by the hypothesis of induction, $D^{\prime}$ is numerically equivalent to a $\mathbb{Q}$-effective $\mathbb{Q}$-Cartier divisor, and hence the assertion follows.

\section{Proof of Proposition 0.5}

Proposition 0.5 is a consequence of Lemma 1.4 and Proposition 3.1 because a pseudo-effective $\mathbb{Q}$-Cartier divisor on an abelian variety is nef.

\section{EXAMPLE 3.2}

Here we show that the $\mathbb{R}$-version of Question 0.2 does not hold in general. Let $k$ be an algebraically closed field. (Note that $k$ is not necessarily an algebraic closure of a finite field.) Let $C$ be an elliptic curve over $k$, and let $A:=C \times C$. Let $\operatorname{NS}(A)$ be the Néron-Severi group of $A$. Note that $\rho:=\operatorname{rk} \operatorname{NS}(A) \geq 3$. By using the Hodge index theorem, we can find a basis $e_{1}, \ldots, e_{\rho}$ of $\mathrm{NS}(A)_{\mathbb{Q}}:=\mathrm{NS}(A) \otimes_{\mathbb{Z}} \mathbb{Q}$ with the following properties:

(a) $e_{1}$ is the class of the divisor $\{0\} \times C+C \times\{0\}$. In particular, $\left(e_{1} \cdot e_{1}\right)=2$.

(b) $\left(e_{i} \cdot e_{i}\right)<0$ for all $i=2, \ldots, \rho$.

(c) $\left(e_{i} \cdot e_{j}\right)=0$ for all $1 \leq i \neq j \leq \rho$.

We set $\lambda_{i}:=-\left(e_{i} \cdot e_{i}\right)$ for $i=2, \ldots, \rho$. Let $\overline{\operatorname{Amp}}(A)$ be the closed cone in $\operatorname{NS}(A)_{\mathbb{R}}:=$ $\mathrm{NS}(A) \otimes_{\mathbb{Z}} \mathbb{R}$ generated by ample $\mathbb{Q}$-Cartier divisors on $A$. It is well known that

$$
\begin{aligned}
\overline{\operatorname{Amp}}(A) & =\left\{\xi \in \mathrm{NS}(A)_{\mathbb{R}} \mid\left(\xi^{2}\right) \geq 0,\left(\xi \cdot e_{1}\right) \geq 0\right\} \\
& =\left\{x_{1} e_{1}+x_{2} e_{2}+\cdots+x_{\rho} e_{\rho} \mid \lambda_{2} x_{2}^{2}+\cdots+\lambda_{\rho} x_{\rho}^{2} \leq 2 x_{1}^{2}, x_{1} \geq 0\right\} .
\end{aligned}
$$

We choose $\left(a_{2}, \ldots, a_{\rho}\right) \in \mathbb{R}^{\rho-1}$ such that

$$
\left(a_{2}, \ldots, a_{\rho}\right) \notin \mathbb{Q}^{\rho-1} \quad \text { and } \quad \lambda_{2} a_{2}^{2}+\cdots+\lambda_{\rho} a_{\rho}^{2}=2 .
$$


Let $E_{i}$ be a $\mathbb{Q}$-Cartier divisor on $A$ such that the class of $E_{i}$ in $\mathrm{NS}(A)_{\mathbb{Q}}$ is equal to $e_{i}$ for $i=1, \ldots, \rho$. If we set $D:=E_{1}+a_{2} E_{2}+\cdots+a_{\rho} E_{\rho}$, then we have the following claim, which is sufficient for our purpose.

\section{CLAIM 3.2.1}

We have that $D$ is nef and $D$ is not numerically equivalent to an effective $\mathbb{R}$ Cartier divisor.

Proof

Clearly $D$ is nef. If we set $e_{1}^{\prime}=e_{1} / \sqrt{2}$ and $e_{i}^{\prime}=e_{i} / \sqrt{\lambda_{i}}$ for $i=2, \ldots, \rho$, then

$$
\overline{\operatorname{Amp}}(A)=\left\{y_{1} e_{1}^{\prime}+y_{2} e_{2}^{\prime}+\cdots+y_{\rho} e_{\rho}^{\prime} \mid y_{2}{ }^{2}+\cdots+y_{\rho}{ }^{2} \leq y_{1}{ }^{2}, y_{1} \geq 0\right\} .
$$

Therefore, as $[D] \in \partial\left(\overline{\operatorname{Amp}}(A)_{\mathbb{R}}\right)$, we can choose

$$
H \in \operatorname{Hom}_{\mathbb{R}}\left(\mathrm{NS}(A)_{\mathbb{R}}, \mathbb{R}\right)
$$

such that

$$
H \geq 0 \text { on } \overline{\operatorname{Amp}}(A) \quad \text { and } \quad\{H=0\} \cap \overline{\operatorname{Amp}}(A)=\mathbb{R}_{\geq 0}[D],
$$

where $[D]$ is the class of $D$ in $\operatorname{NS}(A)_{\mathbb{R}}$. We assume that $D$ is numerically equivalent to an effective $\mathbb{R}$-Cartier divisor $c_{1} \Gamma_{1}+\cdots+c_{r} \Gamma_{r}$, where $c_{1}, \ldots, c_{r} \in \mathbb{R}_{\geq 0}$ and $\Gamma_{1}, \ldots, \Gamma_{r}$ are prime divisors on $A$. As $[D] \neq 0$, we may assume that $c_{1}, \ldots, c_{r} \in$ $\mathbb{R}_{>0}$. Note that $\left[\Gamma_{1}\right], \ldots,\left[\Gamma_{r}\right] \in \overline{\operatorname{Amp}}(A)$ and

$$
0=H([D])=c_{1} H\left(\left[\Gamma_{1}\right]\right)+\cdots+c_{r} H\left(\left[\Gamma_{r}\right]\right),
$$

so that $H\left(\left[\Gamma_{1}\right]\right)=\cdots=H\left(\left[\Gamma_{r}\right]\right)=0$, and hence $\left[\Gamma_{1}\right], \ldots,\left[\Gamma_{r}\right] \in \mathbb{R}_{\geq 0}[D]$. In particular, there is $t \in \mathbb{R}_{\geq 0}$ with $\left[\Gamma_{1}\right]=t[D]$. Here we can set

$$
\left[\Gamma_{1}\right]=b_{1} e_{1}+\cdots+b_{\rho} e_{\rho} \quad\left(b_{1}, \ldots, b_{\rho} \in \mathbb{Q}\right) .
$$

Thus, $b_{1}=t, b_{2}=t a_{2}, \ldots, b_{\rho}=t a_{\rho}$. As $\left[\Gamma_{1}\right] \neq 0, t \in \mathbb{Q}^{\times}$, and hence $\left(a_{2}, \ldots, a_{\rho}\right)=$ $t^{-1}\left(b_{2}, \ldots, b_{\rho}\right) \in \mathbb{Q}^{\rho-1}$. This is a contradiction.

\section{REMARK 3.3}

Let $k$ be an algebraic closure of a finite field, and let $X$ be a normal projective variety over $k$. Let $\mathrm{NS}(X)$ be the Néron-Severi group of $X$, and let $\mathrm{NS}(X)_{\mathbb{R}}:=$ $\mathrm{NS}(X) \otimes_{\mathbb{Z}} \mathbb{R}$. Let $\overline{\mathrm{Eff}}(X)$ be the closed cone in $\mathrm{NS}(X)_{\mathbb{R}}$ generated by pseudoeffective $\mathbb{R}$-Cartier divisors on $X$. We assume that $\overline{\mathrm{Eff}}(X)$ is a rational polyhedral cone; that is, there are pseudo-effective $\mathbb{Q}$-Cartier divisors $D_{1}, \ldots, D_{n}$ on $X$ such that $\overline{\operatorname{Eff}}(X)$ is generated by the classes of $D_{1}, \ldots, D_{n}$. Then the $\mathbb{Q}$-version of Question 0.2 implies the $\mathbb{R}$-version of Question 0.2 .

\section{EXAMPLE 3.4}

This is an example due to Yuan [24]. Let us fix an algebraically closed field $k$ and an integer $g \geq 2$. Let $C$ be a smooth projective curve over $k$, and let $f: X \rightarrow C$ be an abelian scheme over $C$ of relative dimension $g$. Let $L$ be an $f$-ample invertible 
sheaf on $X$ such that $[-1]^{*}(L) \simeq L$ and $L$ is trivial along the zero section of $f: X \rightarrow C$.

CLAIM 3.4.1

(a) $[2]^{*}(L) \simeq L^{\otimes 4}$.

(b) $L$ is nef.

Proof

(a) As $\left.\left.[2]^{*}(L)\right|_{f^{-1}(x)} \simeq L^{\otimes 4}\right|_{f^{-1}(x)}$ for all $x \in C$, there is an invertible sheaf $M$ on $C$ such that $[2]^{*}(L) \simeq L^{\otimes 4} \otimes f^{*}(M)$. Let $Z_{0}$ be the zero section of $f: X \rightarrow C$. Then

$$
\mathcal{O}_{Z_{0}} \simeq[2]^{*}\left(\left.L\right|_{Z_{0}}\right)=\left.\left.[2]^{*}(L)\right|_{Z_{0}} \simeq L^{\otimes 4} \otimes f^{*}(M)\right|_{Z_{0}} \simeq M,
$$

so that we have the assertion.

(b) Let $A$ be an ample invertible sheaf on $C$ such that $L \otimes f^{*}(A)$ is ample. Let $\Delta$ be a horizontal curve on $X$. As $f \circ\left[2^{n}\right]=f$ and $\left[2^{n}\right]^{*}(L) \simeq L^{\otimes 4^{n}}$, by using (a),

$$
0 \leq\left(L \otimes f^{*}(A) \cdot\left[2^{n}\right]_{*}(\Delta)\right)=\left(\left[2^{n}\right]^{*}\left(L \otimes f^{*}(A)\right) \cdot \Delta\right)=\left(L^{\otimes 4^{n}} \otimes f^{*}(A) \cdot \Delta\right),
$$

so that $(L \cdot \Delta) \geq-4^{-n}\left(f^{*}(A) \cdot \Delta\right)$ for all $n>0$. Thus, $(L \cdot \Delta) \geq 0$.

CLAIM 3.4.2

If the characteristic of $k$ is zero and $f$ is nonisotrivial, then $L$ does not have the Dirichlet property (i.e., $L$ is not $\mathbb{Q}$-effective).

\section{Proof}

The following proof is due to Yuan [24]. An alternative proof can be found in [6, Theorem 4.3]. We need to see that $H^{0}\left(X, L^{\otimes n}\right)=0$ for all $n>0$. We set $d_{n}=$ rk $f_{*}\left(L^{\otimes n}\right)$. By changing the base $C$ if necessary, we may assume that all $\left(d_{n}\right)^{2}$ torsion points on the generic fiber $X_{\eta}$ of $f: X \rightarrow C$ are defined over the function field of $C$. By using the algebraic theta theory due to Mumford (especially [19, last line on p. 81]), there is an invertible sheaf $M$ on $C$ such that $f_{*}\left(L^{\otimes n}\right)=M^{\oplus d_{n}}$. On the other hand, by [13],

$$
\operatorname{deg}\left(\operatorname{det}\left(f_{*}\left(L^{\otimes n}\right)\right)^{\otimes 2} \otimes f_{*}\left(\omega_{X / C}\right)^{\otimes d_{n}}\right)=0 ;
$$

that is, $2 \operatorname{deg}(M)+\operatorname{deg}\left(f_{*}\left(\omega_{X / C}\right)\right)=0$. As $f$ is nonisotrivial, we can see that $\operatorname{deg}\left(f_{*}\left(\omega_{X / C}\right)\right)>0$, so that $\operatorname{deg}(M)<0$, and hence the assertion follows.

When the characteristic of $k$ is positive, we do not know the $\mathbb{Q}$-effectivity of $L$ in general. In [15], there is an example with the following properties:

(a) $g=2$ and $C=\mathbb{P}_{k}^{1}$.

(b) There are an abelian surface $A$ over $k$ and an isogeny $h: A \times \mathbb{P}_{k}^{1} \rightarrow X$ over $\mathbb{P}_{k}^{1}$. 


\section{CLAIM 3.4.3}

In the above example, $L$ has the Dirichlet property.

Proof

Replacing $L$ by $L^{\otimes n}$, we may assume that $d:=\operatorname{rk} f_{*}(L)>0$. Let

$$
p_{1}: A \times \mathbb{P}_{k}^{1} \rightarrow A \quad \text { and } \quad p_{2}: A \times \mathbb{P}_{k}^{1} \rightarrow \mathbb{P}_{k}^{1}
$$

be the projections to $A$ and $\mathbb{P}_{k}^{1}$, respectively. Note that $h^{*}(L)$ is symmetric and $h^{*}(L)$ is trivial along the zero section of $p_{2}$. Since $\omega_{A \times \mathbb{P}_{k}^{1} / P_{k}^{1}} \simeq p_{1}^{*}\left(\omega_{A}\right)$, we have that $\left(p_{2}\right)_{*}\left(\omega_{A \times \mathbb{P}_{k}^{1} / P_{k}^{1}}\right) \simeq \mathcal{O}_{\mathbb{P}_{k}^{1}}$, so that, by [13], $\operatorname{deg}\left(\operatorname{det}\left(\left(p_{2}\right)_{*}\left(h^{*}(L)\right)\right)\right)=0$; that is, if we set

$$
\left(p_{2}\right)_{*}\left(h^{*}(L)\right)=\mathcal{O}_{\mathbb{P}_{k}^{1}}\left(a_{1}\right) \oplus \cdots \oplus \mathcal{O}_{\mathbb{P}_{k}^{1}}\left(a_{d}\right),
$$

then $a_{1}+\cdots+a_{d}=0$. Thus, $a_{i} \geq 0$ for some $i$, and hence

$$
H^{0}\left(A \times \mathbb{P}_{k}^{1}, h^{*}(L)\right) \neq 0 .
$$

Therefore, $L$ is $\mathbb{Q}$-effective by Lemma 1.3 .

The above claim suggests that the set of preperiodic points of the map [2]:X $\rightarrow X$ is not dense in the analytification $X_{v}^{\text {an }}$ at any place $v$ of $\mathbb{P}_{k}^{1}$ with respect to the analytic topology (cf. [5]).

Acknowledgments. I would like to thank Professors Biswas, Keel, Langer, Tanaka, and Totaro for their helpful comments. I would especially like to express my hearty thanks to Professor Yuan for his nice example. I would also like to thank the referee for the suggestions.

\section{References}

[1] I. Biswas and Y. I. Holla, Comparison of fundamental group schemes of a projective variety and an ample hypersurface, J. Algebraic Geom. 16 (2007), 547-597. MR 2306280. DOI 10.1090/S1056-3911-07-00449-3.

[2] I. Biswas and A. J. Parameswaran, On vector bundles on curves over $\overline{\mathbb{F}}_{p}, \mathrm{C}$. R. Math. Acad. Sci. Paris 350 (2012), 213-216. MR 2891114.

DOI 10.1016/j.crma.2012.01.006.

[3] I. Biswas and S. Subramanian, On a question of Sean Keel, J. Pure Appl. Algebra, 215 (2011), 2600-2602. MR 2802149. DOI 10.1016/j.jpaa.2011.03.002.

[4] J. I. Burgos Gil, A. Moriwaki, P. Philippon, and M. Sombra, Arithmetic positivity on toric varieties, to appear in J. Algebraic Geom., preprint, arXiv:1210.7692v1 [math.AG].

[5] H. Chen and A. Moriwaki, Algebraic dynamical systems and Dirichlet's unit theorem on arithmetic varieties, Int. Math. Res. Not. IMRN, published electronically 1 May 2015. DOI 10.1093/imrn/rnv097. 
[6] E. Colombo and G. P. Pirola, An intersection result for families of abelian varieties, J. Pure Appl. Algebra 129 (1998), 111-122. MR 1624442.

DOI 10.1016/S0022-4049(97)00093-5.

[7] W. Fulton, Intersection Theory, 2nd ed., Ergeb. Math. Grenzgeb. (3) 2, Springer, Berlin, 1998. MR 1644323. DOI 10.1007/978-1-4612-1700-8.

[8] R. Hartshorne, Ample Subvarieties of Algebraic Varieties, Lecture Notes in Math. 156, Springer, New York, 1970. MR 0282977.

[9] - Algebraic Geometry, Grad. Texts in Math. 52, Springer, New York, 1977. MR 0463157.

[10] S. Keel, Polarized pushouts over finite fields, Comm. Algebra 31 (2003), 3955-3982. MR 2007391. DOI 10.1081/AGB-120022449.

[11] A. Langer, Semistable sheaves in positive characteristic, Ann. of Math. (2) 159 (2004), 251-276. MR 2051393. DOI 10.4007/annals.2004.159.251.

[12] On positivity and semistability of vector bundles in finite and mixed characteristics, J. Ramanujan Math. Soc. 28A (2013), 287-309. MR 3115197.

[13] V. Maillot and D. Rössler, On the determinant bundles of abelian schemes, Compos. Math. 144 (2008), 495-502. MR 2406120.

DOI 10.1112/S0010437X07003235.

[14] V. Mehta and S. Subramanian, Nef line bundles which are not ample, Math. Z. 219 (1995), 235-244. MR 1337219. DOI 10.1007/BF02572363.

[15] L. Moret-Bailly, "Familles de courbes et de variétés abéliennes sur $\mathbb{P}^{1}$ " in Séminaire sur les pinceaux de courbes de genre au moins deux, Astérisque 86, Soc. Math. France, Paris, 1981, 125-140. MR 0642675.

[16] A. Moriwaki, Relative Bogomolov's inequality and the cone of positive divisors on the moduli space of stable curves, J. Amer. Math. Soc. 11 (1998), 569-600. MR 1488349. DOI 10.1090/S0894-0347-98-00261-6.

[17] , Toward Dirichlet's unit theorem on arithmetic varieties, Kyoto J. Math. 53 (2013), 197-259. MR 3049312. DOI 10.1215/21562261-1966116.

[18] - Adelic divisors on arithmetic varieties, to appear in Mem. Amer. Math. Soc., preprint, arXiv:1302.1922v2 [math.AG].

[19] D. Mumford, On the equations defining abelian varieties, II, Invent. Math. 3 (1967), 75-135. MR 0219541.

[20] , Abelian Varieties, Tata Inst. Fundam. Res. Stud. Math. 5, Oxford Univ. Press, London, 1974. MR 0282985.

[21] N. Nakayama, Zariski-decomposition and abundance, MSJ Mem. 14, Math. Soc. Japan, Tokyo, 2004. MR 2104208.

[22] S. Subramanian, Strongly semistable bundles on a curve over a finite field, Arch. Math. (Basel) 89 (2007), 68-72. MR 2322782. DOI 10.1007/s00013-007-1995-8.

[23] B. Totaro, Moving codimension-one subvarieties over finite fields, Amer. J. Math. 131 (2009), 1815-1833. MR 2567508. DOI 10.1353/ajm.0.0088.

[24] X. Yuan, personal communication, December 2013. 
Department of Mathematics, Faculty of Science, Kyoto University, Kyoto, 606-8502, Japan; moriwaki@math.kyoto-u.ac.jp 